\title{
Flow and heat transfer past a permeable stretching/shrinking sheet in $\mathrm{Cu}-\mathrm{Al}$ 203/water hybrid nanofluid
}

\begin{abstract}
Purpose: The purpose of this paper is to study the flow and heat transfer of a hybrid nanofluid, $\mathrm{Cu}-\mathrm{Al} 2 \mathrm{O} 3 /$ water, past a permeable stretching/shrinking sheet. The effects of Brownian motion and thermophoresis are considered here. Design/methodology/approach: Similarity transformations are used to reduce the governing partial differential equations to a system of ordinary (similarity) differential equations. A MATLAB solver called the bvp4c is then used to compute the numerical solutions of equations (12) to (14) subject to the boundary conditions of equation (15). Then, the effects of various physical parameters on the flow and thermal fields of the hybrid nanofluid are analyzed. Findings: Multiple (dual) solutions are found for the basic boundary layer equations. A stability analysis is performed to see which solutions are stable and, therefore, applicable in practice and which are not stable. Besides that, a comparison is made between the hybrid nanofluid and a traditional nanofluid, $\mathrm{Cu}$ /water. The skin friction coefficient and Nusselt number of the hybrid nanofluid are found to be greater than that of the other nanofluid. Thus, the hybrid nanofluid has a higher heat transfer rate than the other nanofluid. However, the increase in the shrinking parameter reduces the velocity of the hybrid nanofluid. Originality/value: The present results are original and new for the study of the flow and heat transfer past a permeable stretching/shrinking sheet in $\mathrm{Cu}-\mathrm{Al} 2 \mathrm{O} 3 /$ water hybrid nanofluid.
\end{abstract}

Keyword: Numerical solution; Stretching/shrinking; Hybrid nanofluid; Cu-AI2O3/water hydrid nanofluid 